\title{
Early estimates of 2016/17 seasonal influenza vaccine effectiveness in primary care in France
}

Cécile Souty ${ }^{1 *}$, Ana-Maria Vilcu ${ }^{1}$, Lisandru Capai ${ }^{1,2}$ Sylvie Van der Werf ${ }^{3,4}$ Martine Valette $^{5,6}$ Thierry Blanchon ${ }^{1}$, Bruno Lina ${ }^{5,6}$, Sylvie Behillil ${ }^{3,4}$, Thomas Hanslik ${ }^{1,7,8}$, Alessandra Falchi ${ }^{2}$.

1. Sorbonne Universités, UPMC Univ Paris 06, INSERM, Institut Pierre Louis d'épidémiologie et de Santé Publique (IPLESP UMRS 1136), F-75012, Paris, France

2. EA7310, Université de Corse, Inserm, France

3. Unité de Génétique Moléculaire des Virus à ARN, UMR 3569 CNRS, Université Paris Diderot SPC, Institut Pasteur, Paris, France

4. CNR des Virus influenzae, Institut Pasteur, Paris, France

5. Laboratoire de Virologie, CNR des virus influenza, Institut des Agents Infectieux, Groupement Hospitalier Nord des HCL, Lyon, France

6. Laboratoire Virpath, CIRI Inserm U1111, CNRS 5308, ENS, UCBL, Faculté de Médecine LYON Est, Université de Lyon, Lyon, France

7. AP-HP, Hôpital Ambroise Paré, service de médecine interne, Boulogne-Billancourt, France

8. UFR des Sciences de la Santé Simone-Veil, Université Versailles Saint Quentin en Yvelines, Versailles, France 


\begin{abstract}
Background: The ongoing 2016/17 influenza epidemic in France is characterized by the circulation of $\mathrm{A}(\mathrm{H} 3 \mathrm{~N} 2)$ viruses, known to cause more severe illness among at risk populations.
\end{abstract}

Objectives: The purpose of our study was to provide early influenza vaccine effectiveness (IVE) estimates for the ongoing influenza epidemic in France and compare these estimates over the six post-pandemic IVE.

Study design: We used clinical and virological data collected in primary care by the French Sentinelles network. IVE in preventing influenza infection was estimated by the test-negative design method. The screening method was used to estimate IVE in preventing medicallyattended influenza-like illness among target groups ( $<65$ year with chronic diseases and $\geq 65$ years) since 2010/11 influenza epidemic.

Results: Early IVE estimates in primary care against influenza A(H3N2) were $48 \%$ (95\% confidence interval (CI): 22 to 66) overall and 39\% (95\% CI: -17 to 69) among elderly (aged 65 and older). In comparison to the last six epidemics, 2016/17 early IVE in preventing influenza-like illness among target groups showed VE estimates higher to those reported during the 2011/12 and 2014/15 epidemics.

Conclusions: The moderate 2016/17 IVE estimates were higher than those estimated during influenza $\mathrm{A}(\mathrm{H} 3 \mathrm{~N} 2)$ epidemics with vaccine mismatch.

\title{
Highlights
}

- 2016/17 influenza epidemic in France is dominated by influenza A(H3N2) viruses

- Early 2016/17 influenza vaccine effectiveness estimates were moderate in France

- Early 2016/17 estimates were higher than in past A(H3N2) epidemics

Keywords: influenza; influenza-like illness; vaccine effectiveness; primary care 


\section{Background}

The 2016/17 ongoing influenza epidemic in France is characterized by the predominant circulation of $\mathrm{A}(\mathrm{H} 3 \mathrm{~N} 2)^{2}$ viruses $(>96 \%)^{1,2}$. Although the $\mathrm{A}(\mathrm{H} 3 \mathrm{~N} 2)$ viruses circulating in 2016/17 were well matched with the vaccine strain A/Hong Kong/4801/2014 ${ }^{3}$, increased disease severity and mortality among the elderly was observed ${ }^{1}$, similarly to the 2011/12 and 2014/15 influenza seasons marked by the circulation of antigenically drifted A(H3N2) viruses 4,5

\section{Objectives}

In this context, we estimated early 2016/17 influenza vaccine effectiveness (IVE) in primary care against laboratory-confirmed $\mathrm{A}(\mathrm{H} 3 \mathrm{~N} 2)$ infection in France. We reported temporal dynamic of IVE in preventing influenza-like illness (ILI) among target groups for vaccination for the last six influenza epidemics, with particular focus on epidemic seasons marked by the circulation of $\mathrm{A}(\mathrm{H} 3 \mathrm{~N} 2)$.

\section{Study design}

As previously described ${ }^{5,6}$, sentinel general practitioners (GPs) of the French Sentinelles network reported through the year ILI cases observed in their practice using the following definition: "sudden onset of fever $>39^{\circ} \mathrm{C}\left(102^{\circ} \mathrm{F}\right)$ with respiratory signs and myalgia" 7 . They collected simultaneously nasopharyngeal swabs along with clinical information in a randomized sample of their ILI patients during the influenza surveillance period. Clinical information concerns at least: date of consultation, age, sex, vaccine status for current seasonal influenza trivalent vaccine (all brands), time since vaccination (more or less than 2 weeks), presence of risk factors (chronic illness). Influenza virus typing and influenza A subtyping were conducted using real-time RT-PCR assays by the French National Influenza Reference Center (CNR, Paris and Lyon) and the laboratory of Virology at the University of Corsica.

All nasopharyngeal specimens collected between $3^{\text {rd }}$ October 2016 (2016w40) and $5^{\text {th }}$ February 2017 (2017w05) (Figure 1) were included in the Test-negative design (TND) study 8, 9 . IVE were estimated as 1 - (odds ratio) x 100 obtained using multivariable logistic regression models with influenza virological result as outcome and vaccination status as main effect, while adjusting for age (eight groups), time of onset of symptoms, presence of a chronic disease and sex. Patients recruited outside the virus circulation period as defined by the ECDC protocol were excluded ${ }^{10}$. 
IVE in preventing medically attended ILI in target groups was estimated by the screening method 5, 11 for the ongoing epidemic and over the 2010/11 to 2015/16 epidemics (http://www.sentiweb.fr ${ }^{12}$ ). Proportions of vaccinated cases were computed among ILI cases reported during the epidemic periods. The proportion of vaccinated subjects among the reference population was obtained from administrative sources ${ }^{13}$. IVE estimates were stratified according to age ( $<65$ years with chronic disease; $\geq 65$ years $){ }^{14}$.

Patients with missing values for any of the variables included in the analysis were excluded, as well as children under six months who are not given the vaccine. Vaccines were considered as potentially effective if administrated at least 2 weeks prior to the symptoms onset. Patients whose vaccination occurred $<2$ weeks prior to symptoms onset were considered as not vaccinated.

\section{Results}

In France, estimated ILI incidence exceeded the epidemic threshold in $2016 \mathrm{w} 50\left(12^{\text {th }}\right.$ to $18^{\text {th }}$ December 2016), peaked on 2017 w03 (16 ${ }^{\text {th }}$ to $22^{\text {th }}$ January 2017) and decreased afterwards. Between 2016w50 and 2017w05, sentinel GPs reported 8,655 ILI cases. Since 2016w40, among the 2,513 swabbed patients, 1,281 (51.0\%) were positive for at least one influenza virus of which 1,240 (96.8\%) were A(H3N2) (Figure 1).

A total of 2,088 swabbed patients (1,135 A(H3N2) cases and 953 controls) were eligible for inclusion in the TND study (Table 1). Adjusted IVE estimates against A(H3N2) were 48\% (95\% confidence interval (CI): 22 to 66 ) among the overall population, $34 \%$ (95\% CI: -6 to 60) among all target groups and 35\% (95\% CI -23 to 66) among elderly (aged $\geq 65$ y) (Table 2).

Early 2016/17 IVE in preventing ILI was estimated 54\% among all target groups (95\% CI: 47 to 60 ) and $47 \%$ among elderly (95\% CI: 38 to 55) (Table 2). The dynamic of estimated IVE in preventing ILI for the six last influenza epidemics is reported in Figure 2.

\section{Discussion}

For the overall population consulting in primary care, our early 2016/17 IVE estimates against influenza $\mathrm{A}(\mathrm{H} 3 \mathrm{~N} 2)$ in France suggest a moderate protection of 48\% (95\% CI: 22 to 66). Among elderly, IVE point estimates against A(H3N2) were slightly lower than the overall estimates, but considering the large CI - due to the small sample size, this difference is not significant. Early 2016/17 IVE estimate in preventing ILI among elderly was higher than 
those reported for the 2011/12 and 2014/15 influenza epidemics marked by the circulation of antigenically drifted influenza $\mathrm{A}(\mathrm{H} 3 \mathrm{~N} 2)$ viruses ${ }^{4,5}$.

These early IVE estimates against A(H3N2) in France were comparable to interim 2016/17 Canadian estimates in the overall population (42\% (95\% CI: 18-59)) ${ }^{15}$, estimates from Finland and Sweden $(30-46 \%)$ in elderly $\left(\geq 65\right.$ y) ${ }^{3}$ and estimates from the I-MOVE consortium in Europe (38.0\% overall (95\% CI : 21.3 to 51.2 ), $25.7 \%$ (95\% CI: 1.5 to 43.9) among target groups and $23.4 \%$ (95\% CI: -15.4 to 49.1$)$ among elderly) ${ }^{16}$.

The $\mathrm{A}(\mathrm{H} 3 \mathrm{~N} 2)$ viruses are known to cause more severe illness than $\mathrm{A}(\mathrm{H} 1 \mathrm{~N} 1) \mathrm{pdm09}$ and/or $\mathrm{B}$ viruses especially in at risk groups, such as elderly ${ }^{17}$. However, in comparison to the past influenza epidemics in France, the ongoing epidemic did not show intense activity in primary care $^{2,5}$. Moreover, early 2016/17 IVE estimates among elderly obtained with the screening method suggested a moderate protection of the vaccine compared to the six post-pandemic epidemics but a higher protection compared to seasons 2011/12 and 2014/15 when circulating $\mathrm{A}(\mathrm{H} 3 \mathrm{~N} 2)$ were antigenically drifted from the vaccine strain ${ }^{4,5}$. The significantly higher early 2016/17 IVE estimates among elderly compared to 2014/15 IVE when no vaccine protection was found for this population ${ }^{5}$, could thus be explained in part by the circulation of $\mathrm{A}(\mathrm{H} 3 \mathrm{~N} 2)$ viruses better matched with the vaccine strain ${ }^{3,18}$.

Some limitations should be considered in interpreting our results. First, the absence of influenza virus characterisations limits the interpretation of IVE estimates. Even if the large majority $(98 \%)$ of circulating viruses in Europe since the beginning of the season were subtype $\mathrm{A}(\mathrm{H} 3 \mathrm{~N} 2)$ and characterization showed an antigenically well matching with the vaccine component, some recently circulating $\mathrm{A}(\mathrm{H} 3 \mathrm{~N} 2)$ viruses continue to be difficult to characterise antigenically by hemagglutination inhibition assay ${ }^{3,19}$. Second, the limited sample size for target groups did not allow estimating accurate IVE among this population. Third, IVE estimates through the screening method could be biased by using a non-specific influenza outcome ${ }^{20}$. However, the use of the same standardized database and methodology over the years ${ }^{5,21}$ allowed comparison of IVE levels over the seven influenza epidemics here compared.

\section{Conclusion}

We report early 2016/17 IVE estimates of $48 \%$ for the 2016/17 influenza A(H3N2) epidemic in France. Even if we were not able to report statistical significant IVE against A(H3N2) among elderly, we described trough the screening method a not significant decrease of IVE 
among this target group. Efforts should be increased to investigate IVE among at risk populations.

\section{Competing interests}

None declared.

\section{Funding}

This project has received funding from the European Union's Horizon 2020 research and innovation programme under grant agreement No 634446 to conduct the study in individuals aged 65 years or more and from Santé Publique France, the national public health agency in France.

\section{Ethical approval}

The protocol was conducted in agreement with the Helsinki declaration. Authorization was obtained from the French Data Protection Agency (CNIL, registration number \#471393).

\section{Acknowledgements}

We thank all GPs and paediatricians participating to the French Sentinelles network. We also thank ECDC who contributed to the funding of the study. 
Table 1. Characteristics of controls and influenza A(H3N2) cases included in the early 2016/17 season influenza vaccine effectiveness analysis, French Sentinelles network, $2^{\text {nd }}$ November $2016-4^{\text {th }}$ February $2017(\mathrm{n}=2,088)$.

\begin{tabular}{|c|c|c|c|}
\hline & & $\begin{array}{l}\text { Controls } \\
(n=953) \\
n(\%)\end{array}$ & $\begin{array}{l}\text { Influenza } A(H 3 N 2) \text { cases } \\
(n=1135) \\
n(\%)\end{array}$ \\
\hline \multicolumn{4}{|c|}{ Age group (years) ${ }^{a}$} \\
\hline & $0-4$ & $324(34.0)$ & $187(16.5)$ \\
\hline & $5-14$ & $121(12.7)$ & $246(21.7)$ \\
\hline & $15-64$ & $449(47.1)$ & $601(53.0)$ \\
\hline & $\geq 65$ & $59(6.2)$ & $101(8.9)$ \\
\hline Females & & $471(49.4)$ & $540(47.6)$ \\
\hline Risk group ${ }^{b}$ & & $158(16.6)$ & $206(18.1)$ \\
\hline $\begin{array}{l}\text { Vaccinated with } \\
\text { trivalent vaccine }\end{array}$ & nal & 75 (7.9) & $87(7.1)$ \\
\hline \multicolumn{4}{|c|}{ Interval onset to swab (days) } \\
\hline & $0-1$ & $552(40.6)$ & $661(58.2)$ \\
\hline & $2-4$ & $350(36.7)$ & $432(38.1)$ \\
\hline & $5-7$ & $51(5.3)$ & $42(3.7)$ \\
\hline & Mean & $1.6^{c}$ & $1.5^{c}$ \\
\hline \multicolumn{4}{|c|}{ Symptoms onset (month) } \\
\hline & Nov 2016 & $232(24.3)$ & $66(5.8)$ \\
\hline & Dec 2016 & $325(34.1)$ & 441 (38.9) \\
\hline & Jan 2017 & $387(40.6)$ & $623(54.9)$ \\
\hline & Fev 2017 & $9(0.9)$ & $5(0.4)$ \\
\hline
\end{tabular}

${ }^{a}$ Since influenza vaccines are not given to children under 6 months old they were excluded from the study

${ }^{\mathrm{b}}$ Age $\geq 65$ y or with chronic condition targeted by the vaccine recommandations in France

${ }^{\mathrm{c}}$ Average interval between onset to swab (in days) 
Table 2. Early estimated of influenza vaccine effectiveness for the 2016/17 influenza epidemic against $\mathrm{A}(\mathrm{H} 3 \mathrm{~N} 2)$ laboratory confirmed influenza by age groups and at risk groups ; and against medically attended influenza-like illness by at risk groups, $5^{\text {th }}$ February 2017 , French Sentinelles network.

\begin{tabular}{|c|c|c|c|c|c|c|c|}
\hline Method & Population group & Cases & $\begin{array}{c}\% \text { Cases } \\
\text { vaccinated }\end{array}$ & Controls & $\begin{array}{l}\% \text { Controls } \\
\text { vaccinated }^{\mathrm{b}}\end{array}$ & $\begin{array}{l}\text { Crude VE \% } \\
(95 \% \mathrm{Cl})\end{array}$ & $\begin{array}{c}\text { Adjusted VE \% } \\
(95 \% \mathrm{Cl})\end{array}$ \\
\hline \multirow{5}{*}{$\begin{array}{l}\text { Test- } \\
\text { negative } \\
\text { design } \\
\text { against } \\
\text { A(H3N2) }\end{array}$} & All & 1135 & $6.8 \%$ & 953 & $7.9 \%$ & 15 (-19 to 39$)$ & $48(22 \text { to } 66)^{c}$ \\
\hline & $0-64$ & 1034 & $3.2 \%$ & 894 & $4.6 \%$ & 31 (-10 to 57$)$ & $49(16 \text { to } 70)^{c}$ \\
\hline & $\begin{array}{l}\text { Overall at risk }{ }^{a} \\
0-64 \text { y with chronic }\end{array}$ & 208 & $31.1 \%$ & 163 & $33.7 \%$ & 11 (-38 to 42$)$ & $34(-6 \text { to } 60)^{\mathrm{d}}$ \\
\hline & disease & 105 & $19.0 \%$ & 99 & $21.2 \%$ & 12 (-74 to 56$)$ & $26(-51 \text { to } 64)^{d}$ \\
\hline & $>=65 \mathrm{y}$ & 103 & $43.7 \%$ & 64 & $53.1 \%$ & 31 (-29 to 63$)$ & $39(-17 \text { to } 69)^{\mathrm{e}}$ \\
\hline \multirow{3}{*}{$\begin{array}{l}\text { Screening } \\
\text { method }\end{array}$} & Overall at risk $^{a}$ & 1006 & $28.5 \%$ & - & & & 54 (47 to 60$)$ \\
\hline & $\begin{array}{l}0-64 \text { y with chronic } \\
\text { disease }\end{array}$ & 352 & $15.9 \%$ & - & $37 \%$ & & 68 (58 to 76 ) \\
\hline & $>=65 \mathrm{y}$ & 654 & $35.3 \%$ & - & $51 \%$ & & 47 (38 to 55$)$ \\
\hline
\end{tabular}

\footnotetext{
${ }^{a}$ Age $\geq 65$ y or with chronic condition targeted by the vaccine recommandations in France

${ }^{b}$ For screening method, "\% controls vaccinated" refers to the proportion of the population vaccinated (from the National Health Insurance System, CNAMTS)

${ }^{\mathrm{c}}$ Adjusted for age (eight groups), presence of at least one chronic disease, sex and week of illness onset

${ }^{d}$ Adjusted for age (eight groups), sex and month of illness onset

${ }^{e}$ Adjusted for sex and month of illness onset
} 
Figure 1. Number of positive influenza-like illness patients swabbed by sentinel physicians who tested positive for at least one influenza virus by types/subtypes and proportion of laboratory confirmed influenza patients swabbed by week, French Sentinelles surveillance network, 3 October 2016 - 5 February 2017 (n =2,513).

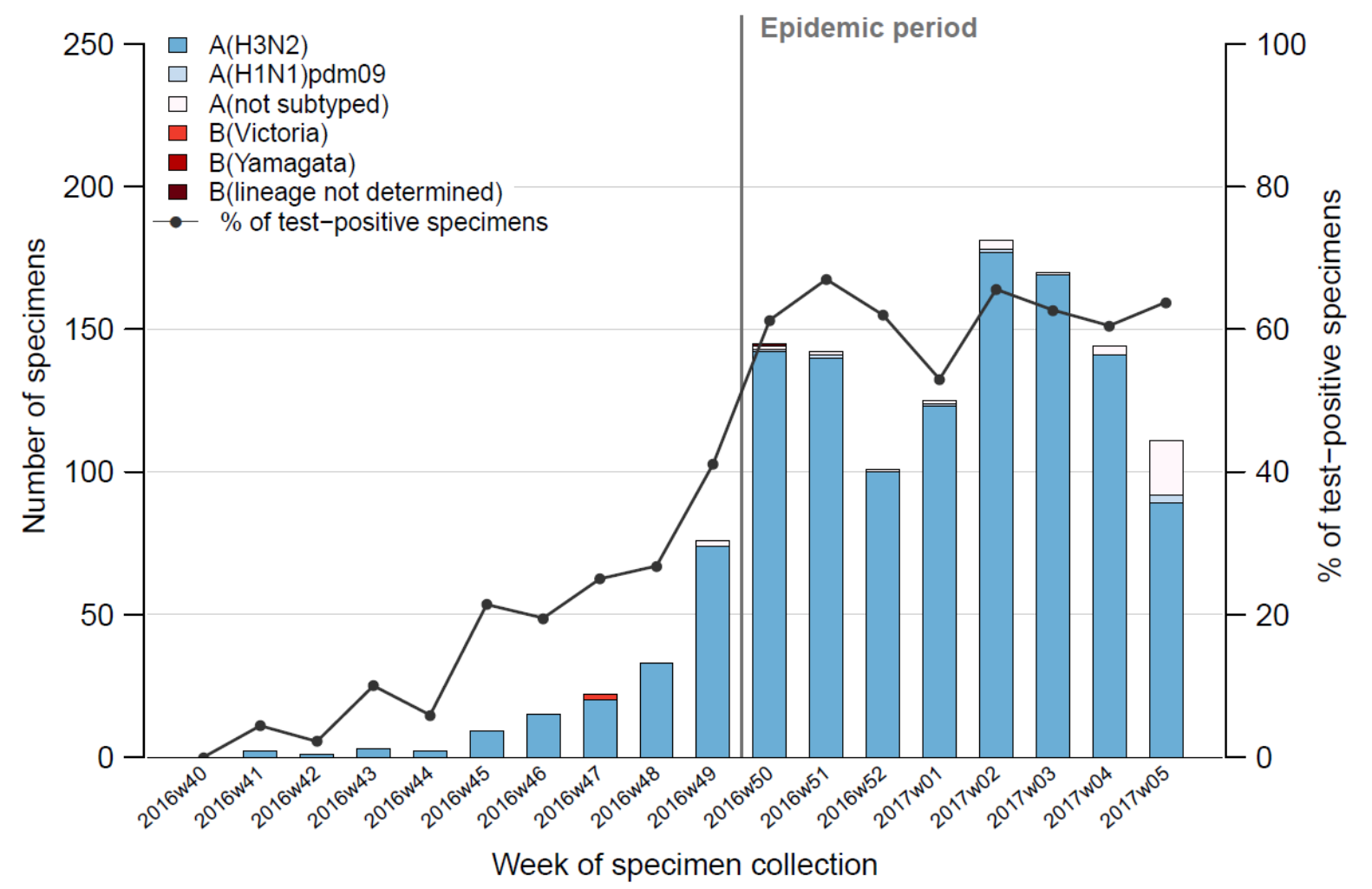


Figure 2. Effectiveness of trivalent seasonal influenza vaccine for 7 influenza epidemics (2010/11 to 2016/17), for at-risk groups (6 months-64 y with chronic illness, over $65 \mathrm{y}$, and overall at risk) estimated by the French Sentinelles surveillance network; segments delimitate the $95 \%$ confidence intervals of the point estimates. For the 2016/17 epidemic, early vaccine effectiveness estimates are reported (from week 2016w50 to week 2017w05).

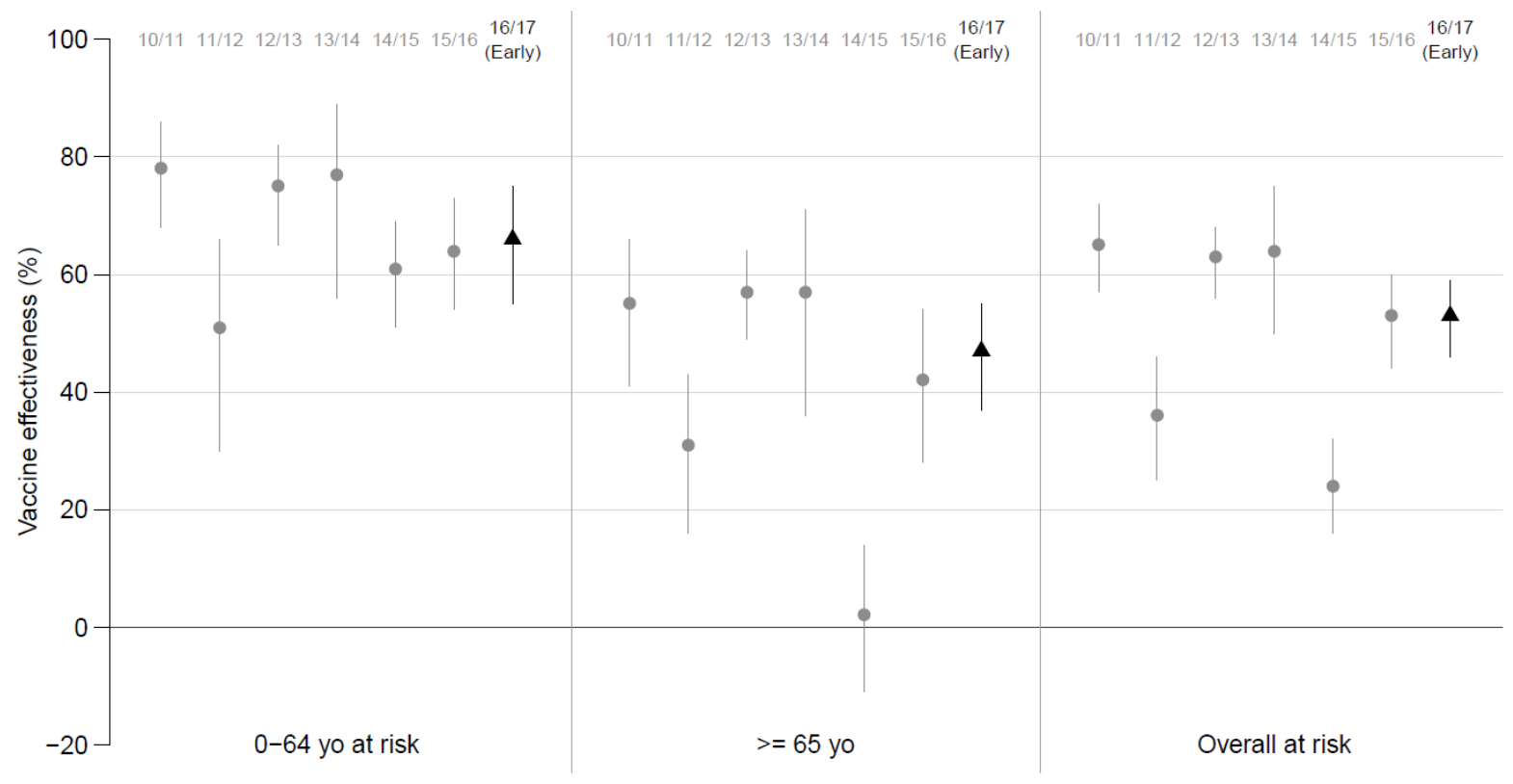




\section{References}

1. Santé Publique France. Bulletin hebdomadaire grippe, semaine 05/2017 - Situation au 08/02/2017. Saint-Maurice: SPF; 2017.

2. French Sentinelles network. Bulletin du réseau Sentinelles du 08/02/17, n 2017s05 (données du 30/01 au 05/02/17). Paris: Inserm-UPMC; 2017.

3. European Centre for Disease Prevention and Control. Risk assessment of seasonal influenza, EU/EEA, 2016-2017 - Update 25 January 2017. Stockholm: ECDC; 2017.

4. Turbelin C, Souty C, Pelat C, Hanslik T, Sarazin M, Blanchon T, et al. Age distribution of influenza like illness cases during post-pandemic $\mathrm{A}(\mathrm{H} 3 \mathrm{~N} 2)$ : comparison with the twelve previous seasons, in France. PLoS One. 2013;8:e65919.

5. Souty C, Blanchon T, Bonmarin I, Lévy-Bruhl D, Behillil S, Enouf V, et al. Early estimates of 2014/15 seasonal influenza vaccine effectiveness in preventing influenza-like illness in general practice using the screening method in France. Hum Vaccin Immunother.

2015;11:1621-25.

6. Falchi A, Turbelin C, Andreoletti L, Arena C, Blanchon T, Bonmarin I, et al. Nationwide surveillance of 18 respiratory viruses in patients with influenza-like illnesses: a pilot feasibility study in the French Sentinel Network. J Med Virol. 2011;83:1451-7.

7. Carrat F, Tachet A, Rouzioux C, Housset B, Valleron AJ. Evaluation of clinical case definitions of influenza: detailed investigation of patients during the 1995-1996 epidemic in France. Clin Infect Dis. 1999;28:283-90.

8. Valenciano M, Ciancio B, Moren A. First steps in the design of a system to monitor vaccine effectiveness during seasonal and pandemic influenza in EU/EEA Member States.

Euro surveillance : bulletin Europeen sur les maladies transmissibles = European communicable disease bulletin. 2008;13.

9. Orenstein WA, Bernier RH, Dondero TJ, Hinman AR, Marks JS, Bart KJ, et al. Field evaluation of vaccine efficacy. Bull World Health Organ. 1985;63:1055-68.

10. European Centre for Disease Prevention and Control. Protocol for case-control studies to measure influenza vaccine effectiveness in the European Union and European Economic Area Member States. Stockholm: ECDC; 2009.

11. Minodier L, Blanchon T, Souty C, Turbelin C, Leccia F, Varesi L, et al. Influenza vaccine effectiveness: best practice and current limitations of the screening method and their implications for the clinic. Expert Rev Vaccines. 2014;13:1039-48.

12. Costagliola D, Flahault A, Galinec D, Garnerin P, Menares J, Valleron AJ. A routine tool for detection and assessment of epidemics of influenza-like syndromes in France. Am J Public Health. 1991;81:97-9.

13. Tuppin P, Choukroun S, Samson S, Weill A, Ricordeau P, Allemand H. Vaccination contre la grippe saisonnière en France en 2010 et 2011 : diminution des taux de couverture et facteurs associés. Presse Med. 2012;41:e568-e76.

14. Farrington CP. Estimation of vaccine effectiveness using the screening method. International journal of epidemiology. 1993;22:742-6.

15. Skowronski DM CC, Sabaiduc S, Dickinson JA, Winter A, De Serres G, Drews SJ, Jassem A, Gubbay JB, Charest H, Balshaw R, Bastien N, Li Y, Krajden M. Interim estimates of 2016/17 vaccine effectiveness against influenza A(H3N2), Canada, January 2017. Euro surveillance : bulletin Europeen sur les maladies transmissibles = European communicable disease bulletin. 2017;22.

16. Kissling E, Rondy M, I-MOVE/I-MOVE+ study team. Early 2016/17 vaccine effectiveness estimates against influenza A(H3N2): I-MOVE multicentre case control studies at primary care and hospital levels in Europe. Euro surveillance : bulletin Europeen sur les maladies transmissibles = European communicable disease bulletin. 2017;22. 
17. Lee CS, Lee JH. Dynamics of clinical symptoms in patients with pandemic influenza A (H1N1). Clin Microbiol Infect. 2010;16:389-90.

18. Darvishian M, Dijkstra F, van Doorn E, Bijlsma MJ, Donker GA, de Lange MMA, et al. Influenza Vaccine Effectiveness in the Netherlands from 2003/2004 through 2013/2014: The Importance of Circulating Influenza Virus Types and Subtypes. PLoS One.

2017; 12:e0169528.

19. European Centre for Disease Prevention and Control. Influenza virus characterisation Summary Europe, September 2016. Stockholm: ECDC; 2016.

20. Orenstein EW, De Serres G, Haber MJ, Shay DK, Bridges CB, Gargiullo P, et al.

Methodologic issues regarding the use of three observational study designs to assess influenza vaccine effectiveness. International journal of epidemiology. 2007;36:623-31.

21. Turbelin C, Boelle PY. Improving general practice based epidemiologic surveillance using desktop clients: the French Sentinel Network experience. Studies in health technology and informatics. 2010;160:442-6. 